\title{
ON ALMOST ORTHOGONALITY IN SIMPLE THEORIES
}

\author{
ITAY BEN-YAACOV AND FRANK O. WAGNER
}

\begin{abstract}
We show that if $p$ is a real type which is internal in a set $\Sigma$ of partial types in a simple theory, then there is a type $p^{\prime}$ interbounded with $p$, which is finitely generated over $\Sigma$, and possesses a fundamental system of solutions relative to $\Sigma$.

2 . If $p$ is a possibly hyperimaginary Lascar strong type, almost $\Sigma$-internal, but almost orthogonal to $\Sigma^{\omega}$, then there is a canonical non-trivial almost hyperdefinable polygroup which multi-acts on $p$ while fixing $\Sigma$ generically. In case $p$ is $\Sigma$-internal and $T$ is stable, this is the binding group of $p$ over $\Sigma$.
\end{abstract}

\section{INTRODUCTION}

In this paper we shall study the interaction of a type $p$ (over some set $A$ in a simple theory) with a family $\Sigma$ of partial types over $A$. Recall that $p$ is

(1) (almost) $\Sigma$-internal if for every realization $a$ of $p$ there are $B \downarrow_{A} a$ and realizations $\bar{c}$ of types in $\Sigma$ over $B$, such that $a \in \operatorname{dcl}(B \bar{c})(\operatorname{resp} . a \in \operatorname{bdd}(B \bar{c}))$.

(2) (almost) generated over $\Sigma$ if there is $B \supseteq A$ such that for any realization $a$ of a $p$ there are realizations $\bar{c}$ of types in $\Sigma$ over $B$ with $a \in \operatorname{dcl}(B \bar{c})$ (resp. $a \in \operatorname{bdd}(B \bar{c}))$.

In a stable theory internality and finite generation are the same, and are an important tool in the analysis of a structure (for instance in Hrushovski's proof that unidimensional stable theories are superstable). Pillay has given examples of simple theories (even of $S U$-rank 1) where they differ [SW02, Examples 2 and 3]. The way out seems to be almost internality and almost generation, as they agree in any simple theory. However, definable as opposed to algebraic closure played an important rôle in the definition of the binding group of $p$ over $\Sigma$, namely the group $\operatorname{Aut}(p / A \cup \Sigma)$ of all permutations of the realizations of $p$ induced by automorphisms fixing $A$ and all realizations of $\Sigma$. If $p$ is $\Sigma$-internal, this group and its action on $p$ are definable in the stable case; moreover the action is transitive if $p$ is a strong type almost orthogonal to $\Sigma$ over $A$. For more details, the reader may consult [Bue96, Section 4.4], [Pil96, Section 7.4], and [Poi87, Section 2.e].

Our Theorem 1.2 improves Theorem 6 of [SW02]. Recall that for $p \in S(A)$ and $\Sigma$ a family of partial types over $A$, a (weak) fundamental system of solutions for $p$ over $\Sigma$ is a tuple $\bar{a}$ of realizations of $p$ such that every realization of $p$ is definable (bounded) over $A \bar{a}$ together with some realizations of $\Sigma$; note that the existence of a (weak) fundamental system of solutions implies (almost) generation. Shami and Wagner show

Date: 22 January 2002.

1991 Mathematics Subject Classification. 03C46.

At the time of the writing of this paper, the first author was a graduate student with the Équipe de Logique Mathématique of Université Paris VII.

The second author would like to thank Bradd Hart for interesting discussions. 
how to obtain, from an almost $\Sigma$-internal real type $p$ in a simple theory, an imaginary type $p^{\prime}$ which has a fundamental system of solutions over $\Sigma$, such that a realization of $p^{\prime}$ is interalgebraic with a finite tuple of realizations of $p$. Theorem 1.2 modifies that argument and obtains such a type $p^{\prime}$ whose realizations are interalgebraic with a single realization of $p$. It follows that Pillay's examples are, in a way, the only ones possible: Up to a finite cover almost internality and generation (even the existence of a fundamental system) are the same in a simple theory.

But obtaining a fundamental system of solutions is not even the main problem. In a stable theory, for every two tuples $a$ and $a^{\prime}$, the following are equivalent:

(1) $a$ and $a^{\prime}$ are conjugate under an automorphism fixing $A \cup \Sigma$ pointwise.

(2) $\operatorname{tp}(a / A \cup \Sigma)=\operatorname{tp}\left(a^{\prime} / A \cup \Sigma\right)$.

(3) $\mathrm{Cb}(a / A \cup \Sigma)=\mathrm{Cb}\left(a^{\prime} / A \cup \Sigma\right)$.

(here we take the canonical base of the type, not of the strong type). However, in the simple case, there is only the implication from top to bottom. The classical definition of the the binding group in a stable theory as $\operatorname{Aut}(p / A \cup \Sigma)$ corresponds to the first condition. This is shown to be unsuitable for the simple case in [SW02], as the group thus obtained can easily be trivialized by adding a generic bipartite graph between $p$ and $\Sigma$, which will not affect independence. The proposed solution, the group Pél $(p, \Sigma)$ of permutations of the realizations of $p$ elementary over $A \cup \Sigma$, corresponds to the second condition (this uses existence of a weak fundamental system of solutions), but it suffers from the same defect (although it is sometimes bigger). In the second part of the paper we shall give a construction corresponding to the third and weakest condition, and therefore to the largest group (or in fact, polygroup). In fact we shall construct a generic poly-chunk multi-acting on $p$ in the sense of [Ben02]. This allows us to invoke the machinery of [BTW, Ben03, TW01] to obtain a coreless almost $A$-hyperdefinable polygroup, or, over some additional parameters, an almost hyperdefinable group acting transitively on an almost hyperdefinable set $X$, whose generic elements are interbounded (over independent parameters) with realizations of $p$. If the theory was stable to start with, the group obtained will be the original binding group.

As usual, we shall fix a complete first-order theory $T$ and work inside a monster model $\mathfrak{C}$; we shall suppose throughout that $T$ is simple. We shall follow the terminology and notation of [Wag00]; in particular the class of a tuple $a$ modulo an equivalence relation $E$ is denoted by $a_{E}$. All types and partial types are hyperimaginary, and tuples can be infinite (of small length), unless stated otherwise. We shall often - and have already done so in the introduction - confound a type $p$, or even a set of partial types $\Sigma$, with the set of its (their) realizations. We shall write $a \equiv_{A} a^{\prime}$ for $\operatorname{tp}(a / A)=\operatorname{tp}\left(a^{\prime} / A\right)$, and $a \equiv_{A}^{L s} a^{\prime}$ for $\operatorname{lstp}(a / A)=\operatorname{lstp}\left(a^{\prime} / A\right)$. If $\left(x_{i}: i<\alpha\right)$ is a sequence, we put $x_{<j}=\left(x_{i}: i<j\right)$ for any $j \leq \alpha$.

\section{From internality to a FUndamental System}

Definition 1.1. Let $\Phi$ and $\Sigma$ be two families of partial types over $A$. The group Pél $(\Phi, \Sigma)$ of elementary permutations of $\Phi$ over $\Sigma$ is the group of all permutations $\tau$ of $\Phi$ such that for all formulas $\varphi(\bar{x}, \bar{y})$ and all $\bar{b} \in \Phi$ and $\bar{a} \in A \cup \Sigma$

$$
\models \varphi(\bar{b}, \bar{a}) \quad \Leftrightarrow \quad \models \varphi(\tau(\bar{b}), \bar{a}) .
$$


Theorem 1.2. Let $T$ be simple, A boundedly closed, and suppose $p=\operatorname{lstp}(a / A)$ is real, and almost internal in a family $\Sigma$ of partial types over $A$. Then there is an imaginary $a^{\prime}$ definable over $A a$, such that a is algebraic over $A a^{\prime}$, and $\operatorname{tp}\left(a^{\prime} / A\right)$ is finitely generated over $\Sigma$. Moreover, $\operatorname{tp}\left(a^{\prime} / A\right)$ has a fundamental system of solutions relative to $\Sigma$.

Proof. By [SW02, Fact 1] (or in fact [Wag00, Proposition 3.4.9]) there is a finite tuple $\bar{a}$ of realizations of $p$ which is a weak fundamental system of solutions for $p$ over $\Sigma$, with uniform algebraicity. Put $\bar{p}=\operatorname{tp}(\bar{a} / A)$, and $G=\operatorname{Pél}(p, \Sigma)$; recall from [SW02] that $G$ is normal in Pél $(p \cup \Sigma, \emptyset)$, and in particular invariant under conjugation by $\operatorname{Aut}(p / A)$.

For any $\bar{b} \models \bar{p}$ let $C(\bar{b})$ be those $g \in G$ which fix $\bar{b}$ pointwise. Recall that two subgroups $H$ and $K$ of a group $G$ are commensurable if their intersection has finite index in either group; a family $\mathfrak{H}$ of subgroups of $G$ is uniformly commensurable if any two $H, K \in \mathfrak{H}$ are commensurable and the index $|H: H \cap K|$ is bounded independently of the choice of $H$ and $K$. Now since any realisation of $p$ is uniformly algebraic over $A \bar{a}$ and a finite tuple of realizations of $\Sigma$, the family $\{C(\bar{b}): \bar{b} \models \bar{p}\}$ is uniformly commensurable. We shall need the following fact:

Fact 1.3. [Sch80, BL89, Wag00, Theorem 4.2.4] Let $G$ be a group and $\mathfrak{H}$ a family of uniformly commensurable subgroups of $G$. Then there is $N \leq G$ which is commensurable with any $H \in \mathfrak{H}$ (necessarily uniformly), and invariant under all automorphisms of $G$ which stabilise $\mathfrak{H}$ setwise. Moreover, $N$ is a finite extension of a finite intersection of groups in $\mathfrak{H}$.

Let $N \leq G$ be this group associated to the family $\{C(\bar{b}): \bar{b} \models \bar{p}\}$; note that the family, and hence $N$, is $\operatorname{Aut}(p / A)$-invariant. Let $a^{\prime}=\{n a: n \in N\}$, an imaginary element. If $\bar{b}=\bar{p}$ and contains $a$, then $|N: N \cap C(\bar{b})|$ is finite, as is $|N: N \cap C(a)|$. It follows that $a^{\prime}$ is a finite set, $\operatorname{Aut}(\mathfrak{C} / A, a)$-invariant, and thus $A a$-definable. Conversely, clearly $a \in \operatorname{acl}\left(a^{\prime}\right)$.

As $a^{\prime} \in \operatorname{dcl}(A a), G$ acts on $p^{\prime}=\operatorname{tp}\left(a^{\prime} / A\right)$ as a group of elementary permutations over $\Sigma$, and we get a homomorphism Pél $(p, \Sigma) \rightarrow \operatorname{Pél}\left(p^{\prime}, \Sigma\right)$. As any two tuples of realizations of $p^{\prime}$ which have the same type over $A \cup \Sigma$ have pre-images with the same type over $A \cup \Sigma$ (since there are only finitely many choices for the pre-image, the fact that $\Sigma$ is large does not matter), this homomorphism is surjective; clearly its kernel contains $N$.

Prolonging $\bar{a}$ if necessary, we may in fact assume that $C(\bar{a}) \leq N$. Let $\bar{a}^{\prime}=\left(a^{\prime}\right.$ : $a \in \bar{a})$. As $C\left(a^{\prime}\right)=N C(a)$, we get $C\left(\bar{a}^{\prime}\right)=N$, so the kernel of the homomorphism is precisely $N$, and $\bar{a}^{\prime}$ is a fundamental system of solutions for $p^{\prime}$ over $\Sigma$.

\section{Almost orthogonality}

Throughout this section we fix a set $\Sigma$ of partial types over $\emptyset$ (or, equivalently, an $\emptyset$-invariant big subclass $\Sigma$ of the monster model $\mathfrak{C}$ ). Recall that it may consist of real, imaginary or hyperimaginary elements.

2.1. Canonical bases. In [HKP00], the existence of canonical bases has been shown for Lascar strong types in a simple theory. In order not to deviate from standard notation, we shall follow the convention that $\operatorname{Cb}(a / b)$ means $\operatorname{Cb}(\operatorname{lstp}(a / b))$, that is $\mathrm{Cb}(\operatorname{tp}(a / \operatorname{bdd}(b)))$, and shall proceed to define a canonical base for a type (as opposed 
to a Lascar strong type). Let us first notice that $\mathrm{Cb}(a / \Sigma)$ is meaningful when $a$ is a tuple of a fixed (small) length, even though $\Sigma$ is not.

Lemma 2.1. Let a be a hyperimaginary.

(1) There exists a tuple $b \subseteq \Sigma$ such that $a \downarrow_{b} \Sigma$.

(2) If $b, b^{\prime} \subseteq \Sigma$ are such that $a \downarrow_{b} \Sigma$ and $a \downarrow_{b^{\prime}} \Sigma$, then $\operatorname{lstp}(a / b)$ and $\operatorname{lstp}\left(a / b^{\prime}\right)$ are parallel (in fact they have a common non-forking extension).

(3) If $b \subseteq \Sigma$ is such that $a \downarrow_{b} \Sigma$, then $\operatorname{Cb}(a / b) \in \operatorname{dcl}(a)$.

ProOF.

(1) By the local character of forking.

(2) As $a \downarrow_{b^{\prime}} b$ and $a \downarrow_{b} b^{\prime}$.

(3) By the previous item, an automorphism fixing $a$ fixes $\mathrm{Cb}(a / b)$.

We may therefore define $\mathrm{Cb}(a / \Sigma)=\mathrm{Cb}(a / b)$ for some (any) $b \subseteq \Sigma$ such that $a \downarrow_{b} \Sigma$. However, $\operatorname{Cb}(a / \Sigma)=\operatorname{Cb}(\operatorname{tp}(a / \operatorname{bdd}(\Sigma)))$, so this will only get us as far as defining the simple analogue of the binding group over bdd $(\Sigma)$.

In a stable theory, $\mathrm{Cb}(a / \Sigma)$ would be the set of canonical parameters for the definition of $\operatorname{tp}(a / \operatorname{bdd}(\Sigma))$, and the set of orbits of such parameters over $\Sigma$ would suffice to define $\operatorname{tp}(a / \Sigma)$. There is a simple analogue:

Lemma 2.2. Let $a$ be a hyperimaginary, and $c=\operatorname{Cb}(a / \Sigma)$. Then there exists $\bar{c} \in$ $\operatorname{dcl}(a) \cap \operatorname{dcl}(\Sigma)$ such that for every automorphism $\sigma$

$$
\sigma(\bar{c})=\bar{c} \Longleftrightarrow \sigma(c) \equiv_{\Sigma} c
$$

One then has $\bar{c} \in \operatorname{dcl}(c)$, $\operatorname{bdd}(c)=\operatorname{bdd}(\bar{c})$, and $a \downarrow_{\bar{c}} \Sigma$.

Proof. We know that $\operatorname{Cb}(a / \Sigma) \in \operatorname{bdd}(\Sigma)$, so there is $b \subseteq \Sigma$ such that $\operatorname{tp}(c / b)$ has a unique extension to $\Sigma$. By [BPW01], the (bounded) set of conjugates of $c$ over $b$ (or equivalently $\Sigma$ ) forms a hyperimaginary $\bar{c} \in \operatorname{dcl}(b)$; clearly $c \in \operatorname{bdd}(\bar{c})$. An automorphism $\sigma$ fixing $a$ fixes also $c$; if $c^{\prime} \equiv_{\Sigma} c$, then $\sigma(\Sigma)=\Sigma$ implies $\sigma\left(c^{\prime}\right) \equiv_{\Sigma}$ $\sigma(c)=c$, whence $\sigma(\bar{c})=\bar{c}$ and $\bar{c} \in \operatorname{dcl}(a)$. Note that this also shows $\bar{c} \in \operatorname{dcl}(c)$. Hence $\operatorname{bdd}(c)=\operatorname{bdd}(\bar{c})$, and $a \downarrow_{c} \Sigma$ implies $a \downarrow_{\bar{c}} \Sigma$.

Notation 2.3. If $a$ and $\bar{c}$ are as above, we write $\bar{c}=\operatorname{Cb}_{\Sigma}(a) . \operatorname{As} \bar{c} \in \operatorname{dcl}(a)$, this is in fact a hyperdefinable function on $\operatorname{tp}(a)$.

When we write $\operatorname{Cb}_{\Sigma}(a)=\operatorname{Cb}_{\Sigma}\left(a^{\prime}\right)$, we understand implicitly that $a \equiv a^{\prime}$.

If $T$ is stable, then $\operatorname{tp}\left(a / \mathrm{Cb}_{\Sigma}(a)\right)$ has a unique non-forking extension to $\Sigma$, and $\mathrm{Cb}_{\Sigma}(a)$ is the canonical base for $\operatorname{tp}(a / \Sigma)$ (the type, not the strong type). In the simple case, we have an independence theorem relative to $\Sigma$ :

Lemma 2.4. Let $a$ and $d$ be any hyperimaginaries, and $b, b^{\prime} \subseteq \operatorname{dcl}(\Sigma)$. Assume that $a \downarrow_{\mathrm{Cb}_{\Sigma}(a)} d, b^{\prime} \downarrow_{\mathrm{Cb}_{\Sigma}(a)} d$ and $b \equiv_{\mathrm{Cb}_{\Sigma}(a)} b^{\prime}$. Then there exists $b^{\prime \prime} \downarrow_{\mathrm{Cb}_{\Sigma}(a)}$ ad realizing $\operatorname{tp}(b / a) \cup \operatorname{lstp}\left(b^{\prime} / \mathrm{Cb}_{\Sigma}(a) d\right)$.

Note that $b \downarrow_{\mathrm{Cb}_{\Sigma}(a)} a$ is automatically true.

Proof. Put $c=\mathrm{Cb}(a / \Sigma)$ and $\bar{c}=\mathrm{Cb}_{\Sigma}(a)$. Then

$$
\bar{c} \in \operatorname{dcl}(c) \cap \operatorname{dcl}(\Sigma) \subseteq \operatorname{dcl}(a),
$$


and $\operatorname{bdd}(c)=\operatorname{bdd}(\bar{c})$. Let $a^{\prime}$ be such that $a b \equiv_{\bar{c}} a^{\prime} b^{\prime}$, and put $c^{\prime}=\operatorname{Cb}\left(a^{\prime} / \Sigma\right)$. Then $c \equiv_{\bar{c}} c^{\prime}$, whereby $c \equiv_{\Sigma} c^{\prime}$, and in particular $c \equiv_{b^{\prime} \bar{c}} c^{\prime}$. Let $a^{\prime \prime}$ be such that $a^{\prime \prime} b^{\prime} c \equiv_{\bar{c}}$ $a^{\prime} b^{\prime} c^{\prime} \equiv_{\bar{c}} a b c$. Then $a^{\prime \prime} \equiv_{c} a$, whence $a^{\prime \prime} \equiv_{c}^{\mathrm{Ls}} a$. Let $\hat{b}$ be such that $a \hat{b} \equiv_{c}^{\mathrm{Ls}} a^{\prime \prime} b^{\prime}$. Then $\hat{b} \equiv_{\bar{c}}^{\mathrm{Ls}} b^{\prime}$ and $\hat{b} \equiv_{a} b$, and we may apply the independence theorem to find $b^{\prime \prime} \downarrow_{\bar{c}} a d$ such that $b^{\prime \prime} \equiv_{a}^{\mathrm{Ls}} \hat{b} \equiv_{a} b$ and $b^{\prime \prime} \equiv_{\bar{c} d}^{\mathrm{Ls}} b^{\prime}$.

Lemma 2.5. For any $x, a$ we have $x \in \operatorname{bdd}(a \Sigma)$ iff $x \in \operatorname{bdd}\left(a, \operatorname{Cb}_{\Sigma}(x a)\right)$.

ProOF. As $x a \downarrow_{\mathrm{Cb}_{\Sigma}(x a)} \Sigma$, we get $x \downarrow_{a, \mathrm{Cb}_{\Sigma}(x a)} \Sigma$; the equivalence follows.

Note that in a stable theory, Lemma 2.5 is true even with bounded replaced by definable closure (see the claim in the proof of Lemma 2.12).

2.2. Getting a poly-chunk. We shall assume familiarity with the theory of generic actions, as developed in [Ben02]. We recall a few of the notions that are used below:

- A partial type $\pi(x)$ over $A$ has definable independence if for any partial type $\pi^{\prime}(y)$ over $A$ the set $\pi(x) \wedge \pi^{\prime}(y) \wedge x \downarrow_{A} y$ is type-definable. (Every complete type has definable independence.)

- A partial type $\pi(x, y, z)$ is an invertible generic action if

(1) $\operatorname{Func}(\pi)=\pi \Gamma_{z}$ and $\operatorname{Arg}(\pi)=\pi \uparrow_{x}$ have definable independence,

(2) $\pi$ implies that $x, y, z$ are pairwise independent,

(3) If $f \models \operatorname{Func}(\pi)$, for any $x$ there are at most boundedly many $y$, and for any $y$ there are at most boundedly many $x$, such that $\models \pi(x, y, f)$. We note $f(a)=\{b: \models \pi(a, b, f)\}$ and $f^{-1}(b)=\{a: \models \pi(a, b, f)\}$.

If $\pi$ is an invertible complete reduced generic action, so is $\pi^{-1}(x, y, z)=$ $\pi(y, x, z)$.

- If $f \models \operatorname{Func}(\pi)$ then the set of its possible germs is

$$
\hat{f}=\{\mathrm{Cb}(a b / f): b \in f(a)\}
$$

If $\pi(x, y, f)$ is a Lascar strong type (we say that $f$ is complete), then its unique germ is denoted by $\bar{f}$.

- The reduction of $\pi(x, y, z)$ is

$$
\bar{\pi}(x, y, \bar{z}):=\exists z[\pi(x, y, z) \wedge \bar{z}=\mathrm{Cb}(x y / z)]
$$

(so in particular, $\bar{z} \in \hat{z}$ ). This is a generic action, whose functions are precisely the germs of functions of $\pi$, whence the notation $\operatorname{Germ}(\pi)=\operatorname{Func}(\bar{\pi})$.

- Two generic actions $\pi(x, y, z)$ and $\pi^{\prime}\left(x^{\prime}, y, z\right)$ are equivalent, denoted $\pi \approx \pi^{\prime}$, if they have the same reduction.

- If $\pi(x, y, z)$ and $\pi^{\prime}\left(y, w, z^{\prime}\right)$ are generic actions, then so is their composition:

$$
\pi^{\prime} \circ \pi\left(x, w, z^{\prime} z\right):=z \downarrow z^{\prime} \wedge x \downarrow z z^{\prime} \wedge \exists y\left[\pi(x, y, z) \wedge \pi^{\prime}\left(y, w, z^{\prime}\right)\right] .
$$

The composition is generic if for every independent $f \in \operatorname{Germ}(\pi)$ and $g \in$ $\operatorname{Germ}\left(\pi^{\prime}\right)$ every $h \in \widehat{g \circ f}$ is independent from each of $f$ and $g$.

Let us return to the problem of constructing an analogue of the binding group. Let $p$ be a Lascar strong type over $\emptyset$ (in other words, $\operatorname{Cb}(p) \in \operatorname{dcl}(\emptyset)$ ), and suppose that $p$ is almost $\Sigma$-internal, but almost orthogonal to $\Sigma(\mathfrak{C})$ (i.e. $x \downarrow \Sigma(\mathfrak{C})$ for every $x \models p$ ). Put

$$
\mathfrak{R}=\{\operatorname{tp}(x, a): x \models p, x \downarrow a, x \in \operatorname{bdd}(a \Sigma)\},
$$


and for any $r(t, u) \in \mathfrak{R}$ define

$$
\pi_{r}\left(t, t^{\prime}, u u^{\prime}\right)=r(t, u) \wedge r\left(t^{\prime}, u^{\prime}\right) \wedge \mathrm{Cb}_{\Sigma}(t u)=\mathrm{Cb}_{\Sigma}\left(t^{\prime} u^{\prime}\right) \wedge u \underset{\mathrm{Cb}_{\Sigma}(t u)}{\downarrow} u^{\prime} .
$$

Lemma 2.6. (1) $\pi_{r}$ is a generic action for every $r \in \mathfrak{R}$, and $\pi_{r}^{-1} \approx \pi_{r}$.

(2) If $\operatorname{tp}\left(x, a^{\prime}\right) \in \mathfrak{R}$, let $\left(x_{i}: i \leq \alpha\right)$ be a Morley sequence in $\operatorname{tp}\left(x / a^{\prime}\right)$ for some infinite ordinal $\alpha$. Then $\operatorname{tp}\left(x_{\alpha}, x_{<\alpha}\right) \in \mathfrak{R}$.

\section{ProOF.}

(1) Assume that $\left(x, y, a_{0} a_{1}\right) \models \pi_{r}$, and put

$$
c=\mathrm{Cb}_{\Sigma}\left(x a_{0}\right)=\mathrm{Cb}_{\Sigma}\left(y a_{1}\right) \quad \text { and } \quad d=\mathrm{Cb}_{\Sigma}\left(a_{0}\right)=\mathrm{Cb}_{\Sigma}\left(a_{1}\right) .
$$

Then $x a_{0} \downarrow_{c} y a_{1}$ and $a_{1} \downarrow_{d} \Sigma$ imply $x a_{0} \downarrow_{d} a_{1}$, whence $x \downarrow_{a_{0}} a_{1}$, and $x \downarrow a_{0} a_{1}$. Since $x \downarrow c$ this also yields $x \downarrow y$. We also have $y \in \operatorname{bdd}\left(a_{1} c\right) \subseteq \operatorname{bdd}\left(x a_{0} a_{1}\right)$. To see that $\pi_{r} \approx \pi_{r}^{-1}$, note that:

$$
\pi_{r}^{-1}\left(t, t^{\prime}, u u^{\prime}\right)=\pi_{r}\left(t^{\prime}, t, u u^{\prime}\right)=\pi_{r}\left(t, t^{\prime}, u^{\prime} u\right) .
$$

Thus every inverse function of $\pi_{r}$ is interdefinable with a function of $\pi_{r}$ which has the same graph, and their germs are therefore equal.

(2) Set $c_{i}=\operatorname{Cb}_{\Sigma}\left(x_{i} a^{\prime}\right) \subseteq \operatorname{dcl}\left(x_{i} a^{\prime}\right)$ for $i \leq \alpha$. Then $\left(x_{i} c_{i}: i \leq \alpha\right)$ is a Morley sequence over $a^{\prime}$, and

$$
\operatorname{Cb}\left(x_{\alpha} c_{\alpha} / a^{\prime}\right) \in \operatorname{dcl}\left(x_{<\alpha} c_{<\alpha}\right) \subseteq \operatorname{bdd}\left(x_{<\alpha} \Sigma\right) .
$$

Then $x_{\alpha} c_{\alpha} \downarrow_{\mathrm{Cb}\left(x_{\alpha} c_{\alpha} / a^{\prime}\right)} a^{\prime}$ implies $x_{\alpha} \downarrow_{c_{\alpha}, \mathrm{Cb}\left(x_{\alpha} c_{\alpha} / a^{\prime}\right)} a^{\prime} c_{\alpha} ;$ as $x_{\alpha} \in \operatorname{bdd}\left(a^{\prime} c_{\alpha}\right)$ by Lemma 2.5, we get

$$
x_{\alpha} \in \operatorname{bdd}\left(c_{\alpha}, \operatorname{Cb}\left(x_{\alpha} c_{\alpha} / a^{\prime}\right)\right) \subseteq \operatorname{bdd}\left(x_{<\alpha} \Sigma\right) .
$$

The next lemma says that if $\models \pi_{r}\left(x, y, a_{0} a_{1}\right)$, then we can replace $r$ with any other $r^{\prime} \in \Re$, and moreover control $\operatorname{lstp}\left(a_{0} / x\right)$.

Lemma 2.7. Let $r, r^{\prime} \in \mathfrak{R}$, and suppose $\models \pi_{r}\left(x, y, a_{0} a_{1}\right)$. If $a^{\prime \prime}$ is such that $\models r^{\prime}\left(x, a^{\prime \prime}\right)$, then there are $a_{0}^{\prime}, a_{1}^{\prime}$ such that:

(1) $\models \pi_{r^{\prime}}\left(x, y, a_{0}^{\prime} a_{1}^{\prime}\right)$.

(2) $\mathrm{Cb}\left(x y / a_{0} a_{1}\right)=\mathrm{Cb}\left(x y / a_{0}^{\prime} a_{1}^{\prime}\right)$.

(3) $a_{0}^{\prime} \equiv_{x}^{\mathrm{Ls}} a^{\prime \prime}$.

Proof. Since we are only interested in $\operatorname{lstp}\left(a^{\prime \prime} / x\right)$ we may assume that $a^{\prime \prime} \downarrow_{x} a_{0}$, whereby $x \downarrow a_{0} a^{\prime \prime}$. As moreover $x \in \operatorname{bdd}\left(a_{0} a^{\prime \prime} \Sigma\right)$, we have

$$
r^{\prime \prime}:=\operatorname{tp}\left(x, a_{0} a^{\prime \prime}\right) \in \mathfrak{R} .
$$

Let $c=\mathrm{Cb}_{\Sigma}\left(x a_{0}\right)=\mathrm{Cb}_{\Sigma}\left(y a_{1}\right)$, and let $C=\mathrm{Cb}_{\Sigma}\left(x a_{0} a^{\prime \prime}\right)$. Note that $c \subseteq C \cap \operatorname{dcl}\left(x a_{0}\right) \cap$ $\operatorname{dcl}\left(y a_{1}\right)$. Since $x a_{0} \equiv_{c} y a_{1}$, we can find $C^{\prime}$ such that $x a_{0} C \equiv_{c} y a_{1} C^{\prime}$. We have $x a_{0} \downarrow_{c} C, y a_{1} \downarrow_{c} C^{\prime}$ and $x a_{0} \downarrow_{c} y a_{1}$. By Lemma 2.4 there is $C^{\prime \prime}$ such that

$$
C^{\prime \prime} \underset{c}{\downarrow} x a_{0} y a_{1} \quad, \quad C^{\prime \prime} \equiv_{y a_{1}} C^{\prime} \quad \text { and } \quad C^{\prime \prime} \equiv_{x a_{0} c}^{\mathrm{Ls}} C .
$$


Hence

$$
x a_{0} \underset{C^{\prime \prime}}{\downarrow} y a_{1} \text { and } y a_{1} C^{\prime \prime} \equiv_{c} y a_{1} C^{\prime} \equiv_{c} x a_{0} C,
$$

So there exist $a_{0}^{\prime}$ and $a_{1}^{\prime}$ such that

$$
\begin{aligned}
& a_{0}^{\prime} C^{\prime \prime} \equiv_{x a_{0} c}^{\mathrm{Ls}} a^{\prime \prime} C \quad \text { with } \quad a_{0}^{\prime} \underset{x a_{0} C^{\prime \prime}}{\downarrow} y a_{1} \quad \text {, and } \\
& y a_{1} a_{1}^{\prime} C^{\prime \prime} \equiv_{c} x a_{0} a^{\prime \prime} C \equiv_{c} x a_{0} a_{0}^{\prime} C^{\prime \prime} \text { with } a_{1}^{\prime} \underset{y a_{1} C^{\prime \prime}}{\perp} x a_{0} a_{0}^{\prime} .
\end{aligned}
$$

Therefore $C^{\prime \prime}=\operatorname{Cb}_{\Sigma}\left(x a_{0} a_{0}^{\prime}\right)=\operatorname{Cb}_{\Sigma}\left(y a_{1} a_{1}^{\prime}\right)$.

By standard independence calculus we obtain that $x a_{0} a_{0}^{\prime} \downarrow_{C^{\prime \prime}} y a_{1} a_{1}^{\prime}$, and conclude that $\models \pi_{r^{\prime \prime}}\left(x, y, a_{0} a_{0}^{\prime} a_{1} a_{1}^{\prime}\right)$.

Let $c^{\prime}=\mathrm{Cb}_{\Sigma}\left(x a_{0}^{\prime}\right)$; since $\mathrm{Cb}_{\Sigma}\left(x a_{0} a_{0}^{\prime}\right)=\mathrm{Cb}_{\Sigma}\left(y a_{1} a_{1}^{\prime}\right)$, we get $c^{\prime}=\mathrm{Cb}_{\Sigma}\left(y a_{1}^{\prime}\right)$ as well. Then $x a_{0}^{\prime} \downarrow_{c^{\prime}} C^{\prime \prime}$ implies $x a_{0}^{\prime} \downarrow_{c^{\prime}} y a_{1}^{\prime}$, so $\models \pi_{r^{\prime}}\left(x, y, a_{0}^{\prime} a_{1}^{\prime}\right)$. As $\pi_{r^{\prime \prime}}$ is a generic action, $x \downarrow a_{0} a_{1} a_{0}^{\prime} a_{1}^{\prime}$, whereby

$$
x y \underset{a_{0}^{\prime} a_{1}^{\prime}}{\downarrow} a_{0} a_{1} \text { and } x y \underset{a_{0} a_{1}}{\downarrow} a_{0}^{\prime} a_{1}^{\prime} .
$$

This implies $\mathrm{Cb}\left(x y / a_{0} a_{1}\right)=\mathrm{Cb}\left(x y / a_{0}^{\prime} a_{1}^{\prime}\right)$.

Finally, $a_{0}^{\prime} \equiv_{x}^{\mathrm{Ls}} a^{\prime \prime}$ holds, as even $a_{0}^{\prime} \equiv_{x a_{0} c}^{\mathrm{Ls}} a^{\prime \prime}$.

Corollary 2.8. We have $\pi_{r} \approx \pi_{r^{\prime}}$ for every $r, r^{\prime} \in \mathfrak{R}$. In other words, the reduction $\bar{\pi}_{r}$ does not depend on $r$.

Let $\pi=\bar{\pi}_{r}$ be this common reduction. Then $\pi=\pi^{-1}$ by Lemma 2.6.1. Now Lemma 2.7 can be restated as:

If $f \in \operatorname{Func}(\pi)$ (i.e., $f \in \operatorname{Germ}\left(\pi_{r}\right)$ for some, or equivalently for every, $r \in \mathfrak{R}$ ) and $y \in f(x)$, then for every $r \in \mathfrak{R}$ there are $a_{0}, a_{1}$ such that $\models \pi_{r}\left(x, y, a_{0} a_{1}\right)$ and $f=\mathrm{Cb}\left(x y / a_{0} a_{1}\right)$. Moreover, $\operatorname{lstp}\left(a_{0} / x\right)$ may be chosen to be any extension of $r(x, u)$ to a Lascar strong type over $x$.

Lemma 2.9. Let $f, g \in \operatorname{Func}(\pi)$ be independent, let $h \in \widehat{g \circ f}$, and let this be witnessed by xyz. Then there are $r \in \mathfrak{R}$ and $\left(a_{i}: i<3\right)$ such that:

(1) $\models r\left(x, a_{0}\right) \wedge r\left(y, a_{1}\right) \wedge r\left(z, a_{2}\right)$.

(2) $\operatorname{Cb}_{\Sigma}\left(x a_{0}\right)=\mathrm{Cb}_{\Sigma}\left(y a_{1}\right)=\mathrm{Cb}_{\Sigma}\left(z a_{2}\right)$.

(3) $\left\{x a_{0}, y a_{1}, z a_{2}\right\}$ are independent over this common canonical base.

(4) $f=\mathrm{Cb}\left(x y / a_{0} a_{1}\right), g=\mathrm{Cb}\left(y z / a_{1} a_{2}\right)$, and $h=\mathrm{Cb}\left(x z / a_{0} a_{2}\right)$.

(5) $\mathrm{fgh}$ is independent of each of the $a_{i}$.

(6) $f, g, h$ are pairwise independent.

Thus in particular,

$$
\models \pi_{r}\left(x, y, a_{0} a_{1}\right) \wedge \pi_{r}\left(x, z, a_{0} a_{2}\right) \wedge \pi_{r}\left(y, z, a_{1} a_{2}\right),
$$

so $h \in \operatorname{Germ}\left(\pi_{r}\right)=\operatorname{Func}(\pi)$.

Moreover, there is $\bar{r}(t, \bar{u}) \in \Re$ such that we can always take $r$ to be the restriction of $\bar{r}$ to $(t, u)$ where $u \subseteq \bar{u}$; and there is a manner to choose $u$ such that if $r(t, u)$ and $r^{\prime}\left(t, u^{\prime}\right)$ were both constructed in this manner then $r \cap r^{\prime}=\bar{r} \uparrow_{\left(t, u \cap u^{\prime}\right)}$ is also in $\mathfrak{R}$ and can serve in place of both $r$ and $r^{\prime}$ (and in fact this holds for any "small" number of $r_{i}$, not only for two). 
Proof. Let $\bar{r}(t, \bar{u}) \in \mathfrak{R}$ be constructed from any type in $\mathfrak{R}$ as in Lemma 2.6.2, where the sequence is of length $\alpha=|T|^{+}$. Then for every infinite subsequence $u^{\prime} \subseteq \bar{u}$ we still have $\bar{r} \uparrow_{\left(t, u^{\prime}\right)} \in \Re$.

By assumption $y \in f(x)$ and $z \in g(y)$. By Lemma 2.7 (restated) $f, g \in \operatorname{Germ}\left(\pi_{\bar{r}}\right)$, and there are $\left(\bar{a}_{i}: i<4\right)$ such that

(1) $\models \pi_{\bar{r}}\left(x, y, \bar{a}_{0} \bar{a}_{1}\right) \wedge \pi_{\bar{r}}\left(y, z, \bar{a}_{2} \bar{a}_{3}\right)$.

(2) $f=\mathrm{Cb}\left(x y / \bar{a}_{0} \bar{a}_{1}\right)$ and $g=\mathrm{Cb}\left(y z / \bar{a}_{2} \bar{a}_{3}\right)$.

(3) $\bar{a}_{1} \equiv_{y}^{\mathrm{Ls}} \bar{a}_{2}$.

As $f, g, h$ are at worst countable hyperimaginaries, there is $\alpha_{0}<|T|^{+}$such that $\operatorname{tp}\left(f g h / \bar{a}_{i}\right)$ does not fork over the first $\alpha_{0}$ elements of the sequence $\bar{a}_{i}$, for all $i<4$. If $a_{i}^{\prime}$ is the sequence of the remaining elements (with indices $\geq \alpha_{0}$ ), then $f g h \downarrow a_{i}^{\prime}$, as $\bar{a}_{i}$ is independent. Let

$$
r=\bar{r}(t, \bar{u})\left\lceil_{\left(t, u_{\geq \alpha_{0}}\right)}=\operatorname{tp}\left(x, a_{0}^{\prime}\right)=\operatorname{tp}\left(y / a_{1}^{\prime}\right)=\operatorname{tp}\left(y / a_{2}^{\prime}\right)=\operatorname{tp}\left(z, a_{3}^{\prime}\right) .\right.
$$

Then $r \in \mathfrak{R}$; as $x \downarrow \bar{a}_{0} \bar{a}_{1}$ implies $x y \downarrow_{a_{0}^{\prime} a_{1}^{\prime}} \bar{a}_{0} \bar{a}_{1}$, and similarly $y z \downarrow_{a_{2}^{\prime} a_{3}^{\prime}} \bar{a}_{2} \bar{a}_{3}$, we get

(1) $\models \pi_{r^{\prime}}\left(x, y, a_{0}^{\prime} a_{1}^{\prime}\right) \wedge \pi_{r^{\prime}}\left(y, z, a_{2}^{\prime} a_{3}^{\prime}\right)$,

(2) $f=\mathrm{Cb}\left(x y / a_{0}^{\prime} a_{1}^{\prime}\right)$ and $g=\mathrm{Cb}\left(y z / a_{2}^{\prime} a_{3}^{\prime}\right)$,

(3) $a_{1}^{\prime} \equiv_{y}^{\mathrm{Ls}} a_{2}^{\prime}$.

Set $d_{0}^{\prime}=\operatorname{Cb}_{\Sigma}\left(a_{0}^{\prime}\right)=\operatorname{Cb}_{\Sigma}\left(a_{1}^{\prime}\right) \in \operatorname{dcl}\left(a_{0}^{\prime}\right) \cap \operatorname{dcl}\left(a_{1}^{\prime}\right) \cap \operatorname{dcl}(\Sigma)$. Then

$$
a_{0}^{\prime} \underset{\operatorname{Cb}_{\Sigma}\left(y a_{1}^{\prime}\right)}{\downarrow} y a_{1}^{\prime} \Longrightarrow a_{0}^{\prime} \underset{d_{0}^{\prime}}{\downarrow} y a_{1}^{\prime} \Longrightarrow a_{0}^{\prime} \underset{a_{1}^{\prime}}{\downarrow} y \Longrightarrow f \underset{a_{1}^{\prime}}{\downarrow} y \Longrightarrow f \downarrow y a_{1}^{\prime} \Longrightarrow x f \underset{y}{\downarrow} a_{1}^{\prime} .
$$

Similarly, we obtain $z g \downarrow_{y} a_{2}^{\prime}$. As $x f \downarrow_{y} z g$ (this is just because $x y z$ witness $h \in \widehat{g \circ f}$ ) and $a_{1}^{\prime} \equiv_{y}^{\mathrm{Ls}} a_{2}^{\prime}$, the independence theorem yields $a_{1}$ with

$$
a_{1} \equiv_{x y f}^{\mathrm{Ls}} a_{1}^{\prime} \quad, \quad a_{1} \equiv_{y z g}^{\mathrm{Ls}} a_{2}^{\prime} \quad \text {, and } \quad a_{1} \underset{y}{\downarrow} x f z g .
$$

Then $x f \downarrow_{y a_{1}} z g$.

Choose $a_{0} \downarrow_{a_{1} x y f} z g$ such that $a_{0} a_{1} \equiv_{x y f} a_{0}^{\prime} a_{1}^{\prime}$, and $a_{2} \downarrow_{a_{1} y z g} a_{0} x f$ such that $a_{1} a_{2} \equiv_{y z g}$ $a_{2}^{\prime} a_{3}^{\prime}$. Then $x f a_{0} \downarrow_{y a_{1}} z g a_{2}$; moreover $f=\mathrm{Cb}\left(x y / a_{0} a_{1}\right)$ and $g=\mathrm{Cb}\left(y z / a_{1} a_{2}\right)$. As $\mathrm{Cb}_{\Sigma}\left(x a_{0}^{\prime}\right)=\mathrm{Cb}_{\Sigma}\left(y a_{1}^{\prime}\right)$ and $\mathrm{Cb}_{\Sigma}\left(y a_{2}^{\prime}\right)=\mathrm{Cb}_{\Sigma}\left(z a_{3}^{\prime}\right)$, we get

$$
\mathrm{Cb}_{\Sigma}\left(x a_{0}\right)=\mathrm{Cb}_{\Sigma}\left(y a_{1}\right)=\mathrm{Cb}_{\Sigma}\left(z a_{2}\right)=: c ;
$$

as $x a_{1}^{\prime} \downarrow_{\mathrm{Cb}_{\Sigma}\left(x a_{1}^{\prime}\right)} y a_{2}^{\prime}$ and $y a_{2}^{\prime} \downarrow_{\mathrm{Cb}_{\Sigma}\left(y a_{2}^{\prime}\right)} z a_{3}^{\prime}$, we see that $\left\{x a_{0}, y a_{1}, z a_{2}\right\}$ are independent over $c$.

Put $d=\operatorname{Cb}_{\Sigma}\left(a_{i}\right) \in \bigcap_{i<3} \operatorname{dcl}\left(a_{i}\right)$. Then $a_{1} \downarrow_{c} a_{2}$ implies $a_{1} a_{2} \downarrow_{d} c$, and

$$
x a_{0} \underset{c}{\downarrow} a_{1} a_{2} \Longrightarrow x a_{0} c \underset{d}{\downarrow} a_{1} a_{2} \Longrightarrow x \underset{a_{0}}{\downarrow} a_{1} a_{2} \Longrightarrow x \downarrow a_{0} a_{1} a_{2} \Longrightarrow x \downarrow f g a_{0} a_{2},
$$

whence $\operatorname{Cb}\left(x z / a_{0} a_{2}\right)=\operatorname{Cb}(x z / f g)=h$.

We saw earlier that $f \downarrow y a_{1}^{\prime}$, whereby $f \downarrow y a_{1}$; by symmetry $f \downarrow x a_{0}, g \downarrow y a_{1}$ and $g \downarrow z a_{2}$. Since we also know that $f a_{0} \downarrow_{y a_{1}} g a_{2}$, independence calculus yields that $f g$ is independent of each of $a_{0}, a_{1}$ and $a_{2}$; since $h \in \operatorname{bdd}(f g)$, the same holds for $f g h$. 
Finally, recall that $f \downarrow a_{0}^{\prime}$, whence $f \downarrow a_{0}$. Hence

$$
a_{1} \underset{c}{\downarrow} a_{0} a_{2} \Longrightarrow a_{1} \underset{d}{\downarrow} a_{0} a_{2} \Longrightarrow a_{1} \underset{a_{0}}{\downarrow} a_{2} \Longrightarrow f \underset{a_{0}}{\downarrow} h \Longrightarrow f \downarrow h
$$

$g \downarrow h$ is proved similarly.

The moreover part is clear by the construction.

We obtain:

Corollary 2.10. The composition $\pi^{2}$ is generic, and $\pi \approx \pi^{2}$ (or equivalently, $\pi_{r}^{2} \approx \pi_{r^{\prime}}$ for any $\left.r, r^{\prime} \in \mathfrak{R}\right)$.

Proof. We saw that whenever $f, g \in \operatorname{Func}(\pi)$ and $h \in \widehat{g \circ f}$, then $f, g, h$ are pairwise independent, which accounts for the genericity of the composition $\pi^{2}$, and that $h \in$ $\operatorname{Func}(\pi)$, whereby $\pi^{2} \approx \pi$.

Theorem 2.11. $\pi$ is a generic poly-chunk in the sense of [Ben02, Definition 3.7], and $\operatorname{Arg}(\pi)=p$. If $P$ is the set of its germs with product given by composition, then $P$ is a polygroup chunk and $\mathrm{SU}(P) \geq \mathrm{SU}(p)$.

Moreover, if $p$ is in a real sort, then $P$ is in a finitary sort (by "real" we mean "real or imaginary", as for us the important distinction is rather from hyperimaginaries).

Proof. By [Ben02, Proposition 2.4 and Theorem 3.9].

For the moreover part, if $x$ is real, then there is a real $a$ such that $r=\operatorname{tp}(x, a) \in \mathfrak{R}$. Assume now that $f$ is a germ of $\pi_{r}$, say $f=\operatorname{Cb}\left(x y / a a^{\prime}\right)$. Then $\operatorname{lstp}\left(x y / a a^{\prime}\right)=$ $\operatorname{tp}\left(x y /\left(\operatorname{dcl}\left(x y a a^{\prime}\right) \cap \operatorname{bdd}\left(a a^{\prime}\right)\right)\right)$, whereby $f \in \operatorname{dcl}\left(x y a a^{\prime}\right)$. As this can be done uniformly, we see that $P$ can be defined in a hyperimaginary sort which is a quotient of $r \times r$.

We can now apply tools from [BTW, Ben03]: Let $\bar{P}$ be the core-reduct of $P$. Then by [BTW], for every $f \in P$ there is a group $G_{f}$ almost hyperdefinable over $f$, whose set of generic elements is the blow-up (along $\bar{f}$ ) of $P^{\prime}$; by [Ben03], there is a unique coreless polygroup $\tilde{P}$, almost hyperdefinable over $\emptyset$, such that $P^{\prime}$ is the set of generic elements of $\tilde{P}$.

Alternatively, we can apply [TW01, Theorem 1.9] and obtain an almost hyperdefinable group $G$ acting transitively and faithfully on an almost hyperdefinable set $X$, such that a generic group element is interbounded over independent parameters with a realization of $\operatorname{Germ}(\pi)$, and a generic element of $X$ is interbounded over independent parameters with a realization of $p$.

2.3. The stable case. Assume now that $T$ is stable, and $p$ is a $\Sigma$-internal strong type over $\emptyset$. We shall show that the polygroup chunk from Theorem 2.11 is the set of generic elements of the usual binding group (in particular composition is unique, and $P$ is a group chunk), and the generic poly-chunk $\pi$ is the generic action of the binding group on $p$.

Lemma 2.12. Let $x_{\leq \omega}$ be a Morley sequence in $p$. Then $x_{\omega} \in \operatorname{dcl}\left(x_{<\omega}, \operatorname{Cb}_{\Sigma}\left(x_{\leq \omega}\right)\right)$.

Proof. Let $y \models p$, and choose $A \downarrow y$ and $a \in \Sigma$ with $y \in \operatorname{dcl}(A a)$. Let $\left(y_{i} a_{i}: i \leq \omega\right)$ be a Morley sequence in $\operatorname{stp}(y a / A)$ with $y_{\omega} a_{\omega}=y a$. Then $\left(y_{i}: i \leq \omega\right)$ is a Morley sequence in $p$; as $p$ is stationary, we may assume $x_{i}=y_{i}$ for $i \leq \omega$. 
Claim. In a stable theory, if $a \in \operatorname{dcl}(b c)$ and $c^{\prime}=\mathrm{Cb}(a b / c)$ (the canonical base of the type, not of the strong type), then $a \in \operatorname{dcl}\left(b c^{\prime}\right)$.

Proof of Claim. Suppose $a^{\prime} \equiv_{b c^{\prime}} a$ with $a^{\prime} \downarrow_{b c^{\prime}} a c$. Then $a^{\prime} b \equiv_{c^{\prime}} a b$ and $a^{\prime} b \downarrow_{c^{\prime}} c$, whence $a^{\prime} b \equiv_{c} a b ;$ as $a \in \operatorname{dcl}(b c)$, we get $a=a^{\prime}$.

It follows that $x \in \operatorname{dcl}(a, \operatorname{Cb}(x a / A))$. But since $\left(x_{i} a_{i}: i \leq \omega\right)$ is a Morley sequence over $A$, we have

$$
\operatorname{Cb}(x a / A) \in \operatorname{dcl}\left(x_{i} a_{i}: i<\omega\right) \subseteq \operatorname{dcl}\left(x_{<\omega} \Sigma\right),
$$

whence $x \in \operatorname{dcl}\left(x_{<\omega} \Sigma\right)$, and $x \in \operatorname{dcl}\left(x_{<\omega}, \operatorname{Cb}_{\Sigma}\left(x_{\leq \omega}\right)\right)$ by the claim again.

Fix $r(t, u)=\operatorname{tp}\left(x_{\omega}, x_{<\omega}\right)$ where $\left(x_{i}: i \leq \omega\right)$ is a Morley sequence in $p$. Note that $r$ is precisely the type constructed in Lemma 2.6.2, and $r \in \mathfrak{R}$.

Recall that a generic function on $p$ is a function $f$ whose domain are the realizations of $p$ independent of $f$ (i.e. of the parameters needed to define $f$ ), and whose values are again independent of $f$. By definability of types in a stable theory, the relation "to agree on an independent realization of $p$ " is a definable equivalence relation for generic functions on $p$, which is a congruence for composition; the equivalence class of $f$ is called the germ of $f$.

Lemma 2.13. $\operatorname{Germ}(\pi)$ is a set of germs of generic functions on $p$, closed under inverse and generic composition.

Proof. If $\models \pi_{r}\left(t, t^{\prime}, u u^{\prime}\right)$, then $\mathrm{Cb}_{\Sigma}(t u) \in \operatorname{dcl}(t u)$, and $t^{\prime} \in \operatorname{dcl}\left(u^{\prime}, \mathrm{Cb}_{\Sigma}\left(t^{\prime} u^{\prime}\right)\right)$ by Lemma 2.12. Hence $t \in \operatorname{dcl}\left(t, u u^{\prime}\right)$; as $t \downarrow u u^{\prime}$ by Lemma 2.6.1, we see that $\operatorname{Func}(\pi)$ is a set of generic functions on $p$ (i.e. $\pi$ is a well-defined generic action on $p$ in the terminology of [Ben02]). We know that $\pi$ is closed under inverse and generic composition; in a stable theory, the reduction of a well-defined generic action is well-defined (i.e. $\operatorname{Germ}(\pi)$ is a set of germs of generic functions on $p$ ), and the composition of well-defined actions is again well-defined and corresponds to the composition of germs.

So the construction gives a generic group chunk which acts generically on $p$. We want to show that this is the generic part of the binding group of $p$ over $\Sigma$. Let $G$ be the binding group of $p$ over $\Sigma$, i.e. the group of permutations of $p$ induced by automorphisms fixing $\Sigma$ pointwise.

Lemma 2.14. Any $g \in G$ induces a germ $\bar{g}$ of a generic function on $p$. If $\left(x_{i}: i<\omega\right)$ is a Morley sequence in $p$ independent of $g$, then $g \in \operatorname{dcl}\left(x_{<\omega}, g\left(x_{<\omega}\right)\right)$.

Proof. If $x \models p$ with $x \downarrow g$, then $g(x) \models p$. But $\operatorname{tp}(g(x))=p=\operatorname{tp}(x), \operatorname{tp}(x / g)$, and $t p(g(x) / g)$ have the same left stratified ranks with respect to that action; as the stratified ranks witness forking, $g(x) \downarrow g$, so $g$ is a generic function on $p$ and induces a germ.

Now suppose $g$ and $g^{\prime}$ have the same type over $x_{<\omega} g\left(x_{<\omega}\right)$. Any $y \models p$ independent of $x_{<\omega}$ is in $\operatorname{dcl}\left(x_{<\omega} \Sigma\right)$; since $g$ and $g^{\prime}$ fix $\Sigma$, we must have $g(y)=g^{\prime}(y)$. Hence $g$ and $g^{\prime}$ agree on all realizations of $p$ independent of $x_{<\omega}$, and thus on any $y \models p$ (just consider a Morley sequence $y_{<\omega}$ independent of $\left.x_{<\omega} g\left(x_{<\omega}\right) y\right)$.

Define

$$
\begin{aligned}
\tilde{\pi}\left(t, t^{\prime}, x_{<\omega} x_{<\omega}^{\prime}\right):= & r\left(t, x_{<\omega}\right) \wedge r\left(t^{\prime}, x_{<\omega}^{\prime}\right) \wedge \mathrm{Cb}_{\Sigma}\left(t x_{<\omega}\right)=\mathrm{Cb}_{\Sigma}\left(t^{\prime} x_{<\omega}^{\prime}\right), \\
& \wedge t \downarrow x_{<\omega} x_{<\omega}^{\prime} \wedge t^{\prime} \downarrow x_{<\omega} x_{<\omega}^{\prime}
\end{aligned}
$$


so $\operatorname{Germ}(\tilde{\pi})$ yields a set of germs of generic functions on $p$. As every $g \in G$ fixes $\Sigma$, we have $\operatorname{Cb}_{\Sigma}\left(x_{<\omega}\right)=\operatorname{Cb}_{\Sigma}\left(g\left(x_{<\omega}\right)\right)$ for any Morley sequence $x_{<\omega}$ in $p$ independent of $g$. Therefore $x_{<\omega} g\left(x_{<\omega}\right) \in \operatorname{Func}(\tilde{\pi})$, and its (unique) germ $\overline{x_{<\omega} g\left(x_{<\omega}\right)}$ does not depend on the choice of $x_{<\omega}$. It follows that

$$
\tau: g \mapsto \overline{x_{<\omega} g\left(x_{<\omega}\right)}
$$

is an embedding preserving multiplication (composition) and inverse. Since the germ $\overline{x_{<\omega} g\left(x_{<\omega}\right)}$ maps any $t \models p$ independent of $x_{<\omega} g\left(x_{<\omega}\right)$ to $g(t)$, the embedding $\tau$ also preserves the action on $p$.

Lemma 2.15. $\tau$ is surjective, and thus a group isomorphism.

Proof. Consider $t t^{\prime} x_{<\omega} x_{<\omega}^{\prime} \models \tilde{\pi}$. Then $t x_{<\omega}$ and $t^{\prime} x_{<\omega}^{\prime}$ have the same type over $\mathrm{Cb}_{\Sigma}\left(t x_{<\omega}\right)$, and hence over $\Sigma$. It follows that there is an automorphism $\sigma$ fixing $\Sigma$ pointwise, and mapping $t x_{<\omega}$ to $t^{\prime} x_{<\omega}^{\prime}$. If $g \in G$ is the element induced by $\sigma$, then $\tau(g)=\overline{x_{<\omega} x_{<\omega}^{\prime}}$.

Lemma 2.16. $\operatorname{Germ}(\pi)$ is a subset of $\operatorname{Germ}(\tilde{\pi})$ containing all generic types.

Proof. $\operatorname{Germ}(\pi)$ is clearly a subset of $\operatorname{Germ}(\tilde{\pi})$ closed under inverse and independent multiplication; we have to show that $\operatorname{Germ}(\pi)^{2}=\operatorname{Germ}(\tilde{\pi})$. But given two Morley sequences $x_{<\omega}$ and $x_{<\omega}^{\prime}$ with $\mathrm{Cb}_{\Sigma}\left(x_{<\omega}\right)=\mathrm{Cb}_{\Sigma}\left(x_{<\omega}^{\prime}\right)$, consider a third Morley sequence $x_{<\omega}^{\prime \prime} \downarrow_{\mathrm{Cb}_{\Sigma}\left(x_{<\omega}\right)} x_{<\omega} x_{<\omega}^{\prime}$ with $\operatorname{Cb}_{\Sigma}\left(x_{<\omega}^{\prime \prime}\right)=\operatorname{Cb}_{\Sigma}\left(x_{<\omega}\right)$. Then $\overline{x_{<\omega} x_{<\omega}^{\prime \prime}}$ and $\overline{x_{<\omega}^{\prime \prime} x_{<\omega}^{\prime}}$ are both in $\operatorname{Germ}(\pi)$, and their composition is $\overline{x_{<\omega} x_{<\omega}^{\prime}}$.

Finally, we note that a generic group chunk is coreless; in this case the group construction of $[\mathrm{BTW}]$ is hyperdefinable (and in fact definable, since equality of germs is definable by stability). The group obtained there is definably isomorphic to the binding group (or to $\operatorname{Germ}(\tilde{\pi})$ ), since a generic group chunk determines its group up to isomorphism.

\section{REFERENCES}

[Ben02] Itay Ben-Yaacov, Group configurations and germs in simple theories, Journal of Symbolic Logic 67 (2002), no. 4, 1581-1600.

[Ben03] _ On the fine structure of the polygroup blow-up, Archive for Mathematical Logic 42 (2003), 649-663.

[BL89] George M. Bergman and Hendrik W. Lenstra, Jr., Subgroups close to normal subgroups, Journal of Algebra 127 (1989), 80-97.

[BPW01] Steven Buechler, Anand Pillay, and Frank O. Wagner, Supersimple theories, Journal of the American Mathematical Society 14 (2001), 109-124.

[BTW] Itay Ben-Yaacov, Ivan Tomašić, and Frank O. Wagner, Constructing an almost hyperdefinable group, preprint.

[Bue96] Steven Buechler, Essential stability theory, Springer Verlag, Berlin, 1996.

[HKP00] Bradd Hart, Byunghan Kim, and Anand Pillay, Coordinatisation and canonical bases in simple theories, Journal of Symbolic Logic 65 (2000), 293-309.

[Pil96] Anand Pillay, Geometric stability theory, Clarendon Press, 1996.

[Poi87] Bruno Poizat, Groupes stables, Nur al-Mantiq wal-Ma'rifah, 1987.

[Sch80] G. Schlichting, Operationen mit periodischen stabilisatoren, Archiv der Mathematik 34 (1980), 97-99.

[SW02] Ziv Shami and Frank O. Wagner, On the binding group in simple theories, Journal of Symbolic Logic 67 (2002), no. 3, 1016-1024. 
[TW01] Ivan Tomašić and Frank O. Wagner, Applications of the group configuration theorem in simple theories, preprint, 2001.

[Wag00] Frank O. Wagner, Simple theories, Kluwer Academic Publishers, 2000.

Itay Ben-YaAcov, Massachusetts Institute of Technology, Department of Mathematics, 77 Massachusetts Avenue, Room 2-101, Cambridge, MA 02139-4307, USA

E-mail address: pezz@math.mit.edu

$U R L:$ http://www-math.mit.edu/ ${ }^{\sim}$ pezz

Frank O. Wagner, Institut Girard Desargues, Université Lyon 1, 21 avenue Claude Bernard, 69622 Villeurbanne cedex, France

E-mail address: wagner@desargues.univ-lyon1.fr 